\section{HIGH FREOUENCY COAXIAL TRANSFORMER WITH FARADAY SHIELD}

\author{
Junwei Lu and Fu Wong
School of Microelectronic Engineering, Faculty of Engineering, \\ Giffith University, Nathan, QLD 4111, AUSTRALIA
}

Abstract - This paper presents the configuration of a high frequency (HF) coaxial transformer with Faraday shield and discusses the shielding effect and insertion loss of the transformer in the high frequency range. The experimental results show the vollage ratio, coupling coefficiency and load characteristic of the HF coaxial transformer in the range up to $2 \mathrm{MHz}$. The numerical result illustrates the eddy current distribution in the windings and shielding coil, and the magnetic flux distribution in the shiclded HF coaxial transformer.

\section{Introduction}

Transformers are often used to provide isolation between two circuits. In these cases the only desirable coupling is that which results from the magnetic field. Actual transformers, not being ideal, have capacitance between the primary and secondary windings, which allows noise coupling through the transformer. This coupling can be eliminated by providing an electrostatic (Faraday) shield which is formed by a ground conductor between the two windings. If properly designed, this shield does not affect the magnetic coupling, but it eliminates the capacitive coupling when the provided shield is grounded. In addition, the shielding coil may contribute some eddy-cument loss in the high frequency range, the location and the thickness of shield should be considered in the design.

\section{Shielded HF Coaxial Transformer}

The insertion loss in the high frequency range above $10 \mathrm{MHz}$, is basically determined by the parasitic capacitances of the windings and the capacitive coupling between the windings. In most applications, a Faraday shield will be required where the high-frequency, high voltage switching waveforms can capacitively couple to the ground plane or secondary output [1].

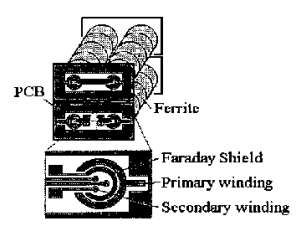

(a)

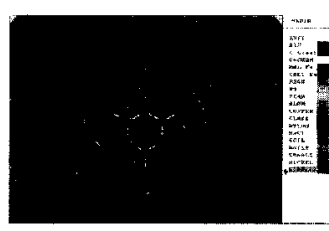

(b)
Fig.1 The shielded IF coaxial transformer, (a) the configuration of the stacked coaxial transformer with turn ratio 1:3, (b) the winding structure and materials used in the structure.
The HF coaxial transformer uses two basic coaxial transformer units stacked together with series connection as shown in Fig. 1 (a). The copper tube is used as a primary winding and the coaxial cable with six wires (two wires parallel connected together) is used as a secondary winding respectively (see Fig. 1 (b)). The grounding line of the corxial cable is used as a shielding coil. The physical size for the ferrite is $20 \mathrm{~mm}$ (outer diameter) $x 10 \mathrm{~mm}$ (inner diameter) $\times 5 \mathrm{~mm}$ (height), the inner and outer diameters of the copper tube are $7 \mathrm{~mm}$ and $8 \mathrm{~mm}$ respectively. The shielding coil has an outer diameter of $3.5 \mathrm{~mm}$ and an inner diameter of $3.1 \mathrm{~mm}$. The experimental results have shown very good performance, with magnetic coupling coefficiency well over $99 \%$, low power loss and high power density.

\section{Eddy-Current Distribution in the Windings and Shielding Coil}

The HF coaxial transformer has many advantages over conventional transformers. It has low eddy-current losses in the windings compared with any other conventional transformer [2]. Fig. 2 (a) shows the eddy-current distribution in both windings and shielding coil, where the excitation source is applied to the primary winding. The secondary winding is short-circuited and the shielding coil located between primary and secondary windings is open-circuited. From the numerical simulation results we can see the eddy current in the shielding coil is rclatively small compared with the eddy-current in the secondary windings, but it contributes some power loss in the transformer. The magnetic flux circulated locally around the each wire of secondary winding has been calculated by numerical method as shown in Fig.2 (b). The highest current density is only located on the surface of the secondary windings.

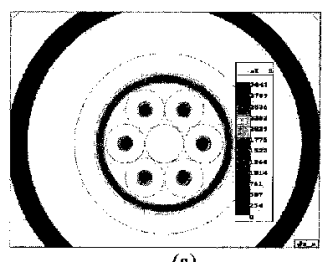

(a)

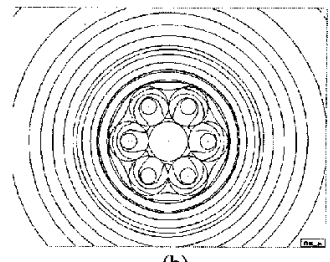

(b)
Fig. 2 Numerical simulation of eddy-current and magnetic flux distributions in the HF coaxial transformer at excitation frequency ( $\mathrm{f}=1 \mathrm{MHz}$ ), (a) eddy-current distribution in both primary and secondary windings, and shielding coil, (b) magnetic flux distribution.

\section{References}

[1] Laszlo Tihanyi, "Electromagnetic Compatibility in Power Electronics", The IEEE Press, 1995

[2] J. Lu, F. P. Dawson and S. Yamada, Application and Analysis of Adjustable Profile High Frequency Switchmode Transformer Having a U-Shaped Winding Structure, IEEE Trans. on Magneties, Vol. 34, NO. 4, pp1345-1347, Jul. 1998 